\title{
Finite-Element Model of Filtration Liquid to a Well in a Deformable Formation
}

\author{
Azhikhanov Nurlan", Zhunisov Nurseit, Marasulov Usen \\ Natural history Faculty, International Kazakh-Turkish University after named Khozha Ahmed Yassawi, Sattarkhan, 161200, \\ Turkestan, Kazakhstan \\ *Corresponding Author: ajihanov1@mail.ru
}

Copyright (C) 2014 Horizon Research Publishing All rights reserved.

\begin{abstract}
In this work we defined the stress-strain state of anisotropic (transversely-isotropic) formation at a liquid filtration in it. There were established connection between voltage of formation and pressure of are filtrated. Numerical solution of the problem was received based on the finite element method with application of an isoparametric element of the first order.
\end{abstract}

Keywords Filtration, Finite Element, Horizontal Well, Deformation, Permeability

\section{Introduction}

A detailed literature review on the research of filtration processes shows that modern technologies and methods of influence on deposits with hardly removable stocks did not find adequate justification of the theory and practice of fluid filtration, taking into account change in structure low productivity of the collector. Also this requires further development of theoretical position on the non-stationary spatial filtering fluids in deformable low productivity porous-fissured, in medium, taking into account the sharp oscillation, hydraulic conductivities and permeability coefficients of energy in multilayer formations, which have the most significant impact on the process of hardly removable stocks. As practice of operation of multi sheeted deposits, an increase in oil production by improving of technology of production of reserves from low-productive formations, tantamount opens a new hydrocarbon fields.

\section{Materials and Methods}

\subsection{Statement of the Problem}

Mountain pressure upon formation is compensated as voltages solid skeleton of array, and fluid pressure. Change of the last perturbs tensely-deformed state of formation
(VAT), i.e. changing of the pore pressure at one point causes reorganization of all the VAT of system and including the deformation throughout the formation.

When in the study of the stress-strain state of the formation is assumed that under the influence of the applied external forces, deformation of the formation proceeds without violation of its continuity [1]. Therefore it is necessary to impose restrictions on size component of deformation.

We will consider an elastic static condition of the horizontal well, the longitudinal axis of which is an arbitrary angle with the line of the strike plan of an isotropy of the rock massiv (fig. 1).

We introduce the rectangular cartesian coordinate system Oxyz in such a way that the axis is Oz directed vertically up, horizontal axis $\mathrm{Ox}$ and $\mathrm{Oy}$ coincide with the lines accordingly transverse to and along the strike plane of isotropy.

Elastic condition of transversaly-isotropic of array described by the equation of generalized Hooke's law in the coordinate system of the $\mathrm{Ox}^{\prime} \mathrm{y}^{\prime} \mathrm{z}^{\prime}$ obtained by turning of Oxyz on angle $\phi$ around the vertical axis Oz and has the form [2]

$$
\{\varepsilon\}=[D]\{\sigma\}
$$

where,

$$
\{\sigma\}=\left\{\begin{array}{l}
\sigma_{x} \\
\sigma_{y} \\
\sigma_{z} \\
\tau_{y z} \\
\tau_{x z} \\
\tau_{x y}
\end{array}\right\}-\text { stress components; }
$$

$$
\{\varepsilon\}=\left\{\begin{array}{l}
\varepsilon_{x} \\
\varepsilon_{y} \\
\varepsilon_{z} \\
\gamma_{y z} \\
\gamma_{x z} \\
\gamma_{x y}
\end{array}\right\}-\text { elastic deformation; }
$$




$$
\begin{gathered}
\left.\frac{\partial P}{\partial z}\right|_{z= \pm L_{z}}=0 ;\left.\quad P\right|_{x= \pm L_{x}}=P_{k_{1}},\left.\quad P\right|_{y= \pm L_{y}}=P_{k_{2}} \\
{[D]=\left[d_{i j}\right](i, j=\overline{1,6})-\text { coefficient of permeability. }}
\end{gathered}
$$

Here coefficients of deformation $d_{i j}$ are defined from [3].

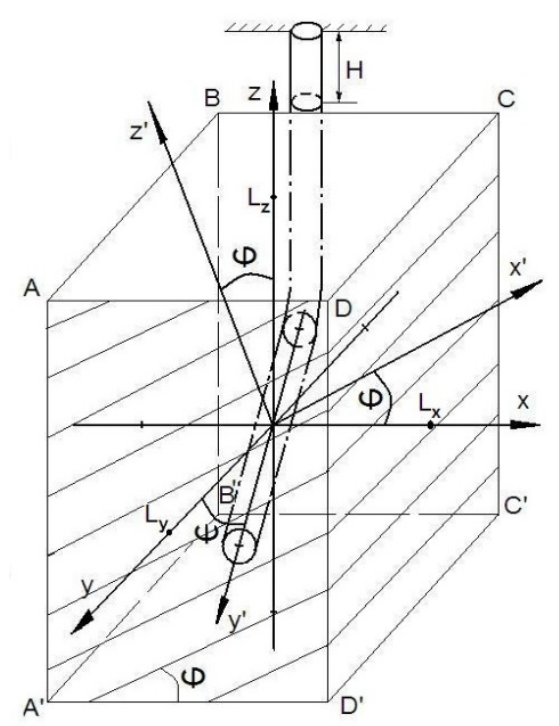

Figure 1. Estimated scheme of the inclined transversally isotropic array with horizontal well

Components of deformation can also be determined through displacement $u, v$ and $w$, (by the Ox, Oy and $\mathrm{Oz}$ axis respectively) by means ratio of Cauchy. Boundary conditions we will set in the form of

$$
\begin{gathered}
u=v=0, \text { when } \\
(x, y, z) \in A B B^{\prime}, B C C^{\prime}, D C C^{\prime} D^{\prime}, A D D^{\prime} A^{\prime}, \\
u=v=w=0 \text { when }(x, y, z) \in A^{\prime} B^{\prime} C^{\prime} D^{\prime} .
\end{gathered}
$$

Next non-stationary filtration of liquid to a horizontal well in transversely isotropic porous medium (Fig. 1) described by the following equation governing the pressure $\mathrm{P}$ spatial filtering

$$
\frac{1}{\eta} \frac{\partial p}{\partial t}-\alpha \frac{\partial \varepsilon_{v}}{\partial t}=\sum_{i=1}^{3} \frac{\partial}{\partial x_{i}}\left(\frac{k_{i j}}{\mu} \frac{\partial p}{\partial x_{j}}\right),
$$

where $\alpha$ - coefficient of discharge; $\eta$-module Bio; $\mu$ - fluid viscosity; $\varepsilon_{\mathrm{v}}$ - volume strain breed; $k_{i j}(i, j=1,2,3)$ - is the permeability coefficients of anisotropic (transversely-isotropic) of the formation.

With boundary conditions

where $P_{k_{1}}, P_{k_{2}}-$ pressures in boundaries; $L_{x}, L_{y}, L_{z}-$ length of the $\mathrm{Ox}, \mathrm{Oy}, \mathrm{Oz}$ axis respectively.

Thus full voltage [4] transversely-isotropic of the formations can be expressed through the effective stress and pressure in the form of

$$
\begin{gathered}
\sigma_{x}^{\Pi}=\sigma_{x}+P, \quad \tau_{x y}^{\Pi}=\tau_{x y}, \quad \tau_{x z}^{\Pi}=\tau_{x z}, \\
\sigma_{y}^{\Pi}=\sigma_{y}+P, \quad \tau_{y z}^{\Pi}=\tau_{y z}, \\
\sigma_{z}^{\Pi}=\sigma_{z}+P .
\end{gathered}
$$

A characteristic feature of the model is the assumption that the porous matrix deformable absolutely freely to up some hard limit $\varepsilon_{0}$.

\subsection{Finite-Element Model}

Consider the three-dimensional isoparametric six-sided finite element first order [1], for which the interpolation polynomial is a linear function of local coordinates (Fig. 2). Limits of change of local coordinates for all elements is $|\xi| \leq 1,|\eta| \leq 1,|\zeta| \leq 1$. Functions forms such as element are given of the form

$$
N_{i}(\xi, \eta, \zeta)=\frac{1}{8}\left(1-\xi \xi_{i}\right)\left(1+\eta \eta_{i}\right)\left(1+\zeta \zeta_{i}\right),
$$

where $\xi_{i}, \eta_{i}, \zeta_{i}-$ local coordinates of a nodal point with number $i,(i=1,2, \ldots, 8)$.
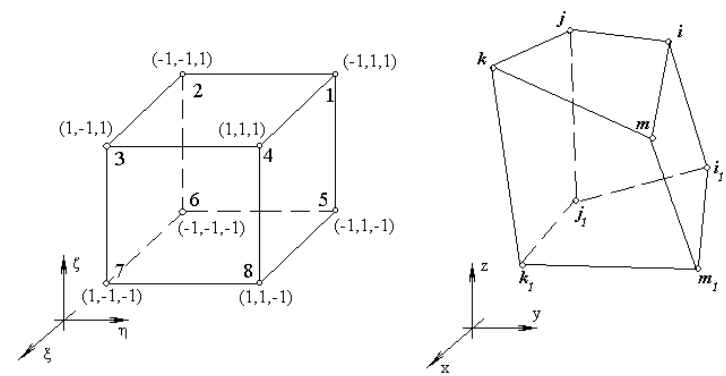

Figure 2. Three-dimensional isoparametrical element

An interpolation relation for displacement in a three-dimensional isoparametrical element has the form

$$
\left[\begin{array}{l}
u(\xi, \eta, \zeta) \\
v(\xi, \eta, \zeta) \\
w(\xi, \eta, \zeta)
\end{array}\right]^{(e)}=\sum_{\alpha=1}^{8} \mathrm{E}_{3} N_{\alpha}\left[\begin{array}{l}
u_{\alpha} \\
v_{\alpha} \\
w_{\alpha}
\end{array}\right]^{(e)},
$$

where $u_{\alpha}, v_{\alpha}, w_{\alpha}$-the components of the displacement vector at the nodes " $e$ "-the element, $(\alpha=1,2, \ldots, 8)$.

$$
E_{3}=\left(\begin{array}{lll}
1 & 0 & 0 \\
0 & 1 & 0 \\
0 & 0 & 1
\end{array}\right) \text {-identity matrix }
$$

Interpolation relations for finite element defined as

$$
\left[\begin{array}{l}
x(\xi, \eta, \zeta) \\
y(\xi, \eta, \zeta) \\
z(\xi, \eta, \zeta)
\end{array}\right]^{(e)}=\sum_{\alpha=1}^{8} \mathrm{E}_{3} N_{\alpha}\left[\begin{array}{l}
x_{\alpha} \\
y_{\alpha} \\
z_{\alpha}
\end{array}\right]^{(e)}
$$


here $x_{\alpha}, y_{\alpha}, z_{\alpha}$ - the global coordinates nodal point with number $\alpha$.

These ratios can be represented as

$$
\begin{aligned}
& x(\xi, \eta, \zeta)=N^{(e)}(\xi, \eta, \zeta) X^{e)} ; \\
& y(\xi, \eta, \zeta)=N^{(e)}(\xi, \eta, \zeta) Y^{(e)} ; \\
& z(\xi, \eta, \zeta)=N^{(e)}(\xi, \eta, \zeta) Z^{(e)} ;
\end{aligned}
$$

where $N^{(e)}$-matrix of function the form of element, $X^{(e)}, Y^{(e)}, Z^{(e)}$ - vectors of nodal values of the global coordinate.

The matrix of rigidity of isoparametrical finite element has the form:

$$
K_{\alpha \beta}^{(e)}=\int_{\Omega^{(e)}} B_{\alpha}^{(e)^{\prime}} D B_{\beta}^{(e)} d \Omega \quad(\alpha, \beta,=1,2, \ldots, 8)
$$

Here the matrix of gradients is $B_{\alpha}^{(e)}=M E_{3} N_{\alpha}$ defined from form function $N_{\alpha}$.

As a result, a matrix of rigidity of an isoparametrical element we get from:

$$
K_{\alpha \beta}^{(e)}=\int_{-1}^{1} \int_{-1}^{1} \int_{-1}^{1} B_{\alpha}^{(e)^{\prime}} D B_{\beta}^{(e)} \operatorname{det} J d \xi d \eta d \zeta
$$

Numerical integration which is performed by Gauss method, i.e.

$$
K_{\alpha \beta}^{(e)}=\left.\sum_{i=1}^{3} \sum_{j=1}^{3} \sum_{k=1}^{3} B_{\alpha}^{(e)^{\prime}} D B_{\alpha}^{(e)} \operatorname{det} J\right|_{\xi_{i}, \eta_{j}, \zeta_{k}} H_{i} H_{j} H_{k}
$$

here $\xi_{i}, \eta_{j}, \zeta_{k}$-points of an integrating of Gauss; J-Jacobi matrix; $n$ - the order of integration; $H_{i}, H_{j}, H_{k}$-weight coefficients.

We define shift of point of an element $u^{(e)}=N^{(e)} U$. The matrix of finite deformation has the form $\varepsilon^{(e)}=B^{(e)} u^{(e)}$, in turn components of tension are computed from $\sigma^{(e)}=D \varepsilon^{(e)}$.

Required values are defined from the decision of systems of he linear algebraic equations [5]

$$
\begin{gathered}
K U=F, \\
K=\sum_{e} a^{(e)^{\prime}} K^{(e)} a^{(e)},
\end{gathered}
$$

here

$$
F=\sum_{e} a^{(e)^{\prime}}\left(F_{\varepsilon_{o}}^{(e)}+F_{\underline{p}}^{(e)}+F_{p}^{(e)}\right)
$$

where $F_{\varepsilon_{O}}^{(e)}, F_{\underline{p}}^{(e)}, F_{\bar{p}}^{(e)}$ - vectors of nodal forces finite element, statically equivalent action of a finite deformation, $\underline{p}$ surface and $p$ volume forces.

$$
\begin{gathered}
F_{\varepsilon_{o}}^{(e)}=\int_{v^{e}} B^{(e)^{\prime}} D \varepsilon_{0} d v, \\
F_{\underline{p}}^{(e)}=\int_{S^{e}} N^{(e)} \underline{p}^{(e)} d s, \\
F_{\bar{p}}^{(e)}=\int_{v^{e}} N^{(e)^{\prime}} G^{(e)} d v .
\end{gathered}
$$

The of an element matrix of stiffness considered above can be written as

$$
K^{(e)}=\int_{v^{e}} B^{(e)^{\prime}} D B^{(e)} d v
$$

By similar way we apply procedure to a six-sided isoparametrical element (fig. 2) to the solution of a problem of a filtration

\subsection{Computing Experiment}

The deformable state inclined at an angle $\varphi$ the transversal-isotropic massif is executed by using of law Hooke and deformation coefficients. Numerical experiment was conducted on the following data: in the capacity of breeds of inclined a layer are taken [2]. Mudstone, siltstone, sandstone, limestone module of elasticity which has respectively $E=1,34 ; 0,62 ; 2,95 ; 5\left(10^{-5} \mathrm{~kg} / \mathrm{cm}^{2}\right)$ constant Poisson respectively $v^{(k)}=0,3 ; 0,2 ; 0,35 ; 0,11$. For transversal -isotropic layers rounded elastic characteristics are determined $E_{1}=1,54 \cdot 10^{-5} \mathrm{~kg} / \mathrm{cm}^{2}$, $E_{2}=0,98 \cdot 10^{-5} \mathrm{~kg} / \mathrm{cm}^{2}$, the modulus shear $G_{2}=0,36 \cdot 10^{-5} \mathrm{~kg} / \mathrm{cm}^{2} ; \quad v_{1}=0,22 \quad, \quad v_{1}=0,25$ Concurrently boundary conditions are considered when determining voltage under $\psi=30^{0}$ in inclined formationon $X$ and $Z$. The comparative isoline shown in Figure 3 in cases $\varphi=30^{0}$ (dotted lines) and $\varphi=60^{0}$ (solid line). General view of the deformation function is presented in Figure 4.

There were conducted various embodiments of calculation depending on the angles of inclination of the plane isotropy and horizontal wells $\varphi, \psi$. 


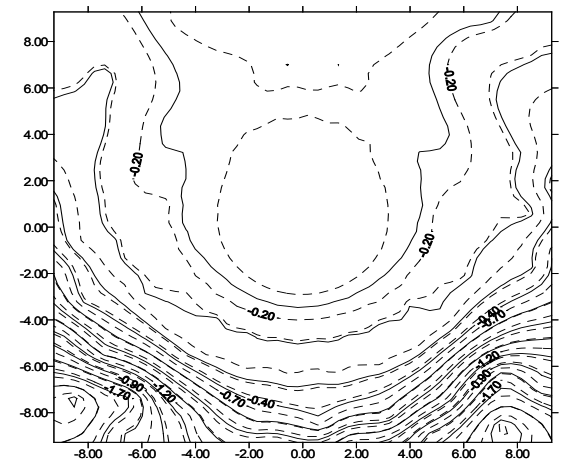

a)

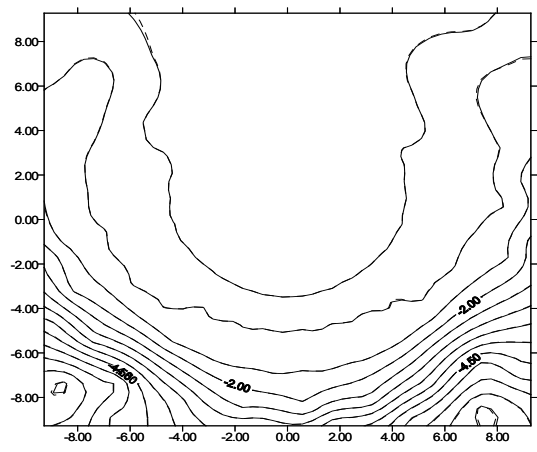

b)

Figure 3. Isoline of the normal tension a) $\sigma_{x}$; b) $\sigma_{z}$

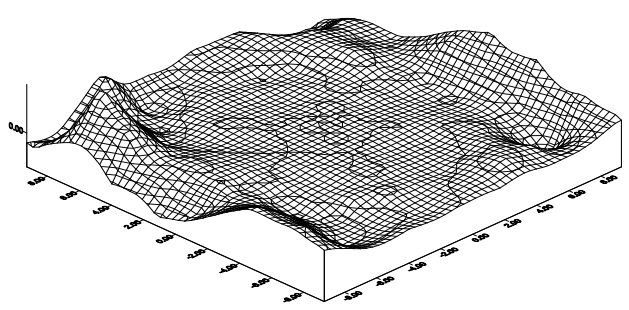

a)

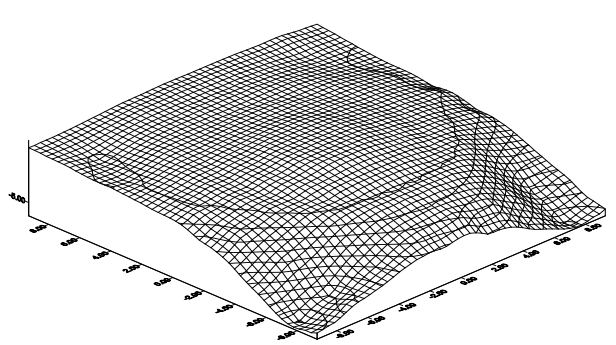

b)

Figure 4. Functions of the components of deformation a) $\varepsilon_{x}$;b) $\varepsilon_{z}$
The analysis of the results given in inclined transversal-isotropic of formation, shows that with increase in quantity of final elements in discrete model of a body, is observed coincidence of two significant figures in values of a component of movement of $\mathrm{u}$, normal tension $\sigma$, and also in values of intensity of tension and deformations.

\section{Conclusions}

In this paper we defined the stress-strain state of anisotropic (transversely-isotropic) formation at a liquid filtration in it. There were established connection between voltage of formation and pressure of are filtrated. Numerical solution of the problem was received based on the FEM with application of an isoparametric element of the first order. the Presented by a finite-element model of voltage deformable condition of formation with horizontal well an arbitrary profile. There were analysed ways of determining the elastic and filtration properties of deformable heterogeneous reservoirs. Thus, by using the finite element model can obtain the change in fluid pressure in the voltage-strain state of a transversely-isotropic formation.

\section{Acknowledgements}

We are very grateful to experts for their appropriate and constructive suggestions to improve this template.

\section{REFERENCES}

[1] Shestakov B.M. Hydrogeomechanica. - Moscow university, 1998. $-72 p$.

[2] Erzhanov Zh.S., Aytaliev Sh.M., Masanov Zh.K. Stability of horizontal tonnels in an obliquely layered array. Almaty, Nauka, 1971, 160p.

[3] Zhumagulov B.T., Masanov Zh.K., Azhikhanov N.T. Calculation of production of horizontal well of type stretch in the anisotropic porous medium // Journal of Mathematics and Technology, ISSN: 2078-0257, February, 2010. P.86-90

[4] Phadeev A.B. Finite element method in the geomechanic. Moscow, Nedra, 1987.-221p.

[5] Borja R., Chao Li. Finite Element Formulation of Poro-Elasticity Suitable for Large Deformation Dynamic Analysis. // Proceedings, Third UJNR Workshop on Soil-Structure Interaction, March 29-30, 2004. -9p 